\title{
Malignant Inferior Vena Cava Neoplasm
}

National Cancer Institute

\section{Source}

National Cancer Institute. Malignant Inferior Vena Cava Neoplasm. NCI Thesaurus. Code C5377.

A malignant neoplasm involving the inferior vena cava. 